\title{
THE DISTRIBUTION OF ANGULAR VELOCITIES AMONG DIATOMIC GAS MOLECULES.
}

\author{
By EDwin C. Kemble.
}

\section{INTRODUCTION.}

\section{$\mathrm{T}^{\mathrm{H}}$}

HE explanation of the structure of the infra-red absorption bands of gases given by Bjerrum ${ }^{1}$ has led to a striking direct confirmation of the quantum theory in the form first proposed by Planck (assuming absorption as well as emission by quanta), and gives to the study of these bands a large significance for the further development of that theory. As this paper and the one which follows it are based on Bjerrum's ideas, a brief summary of the theory is given for the benefit of readers who may not have followed the original German papers containing its statement and the subsequent confirmation of its predictions.

As early as 1904 Drude, ${ }^{2}$ from the study of the dispersion of various crystals, was led to the conclusion that the infra-red absorption and emission bands of most substances (including gases) must be due to the vibrations of electrically charged atoms and molecules rather than to the oscillations of the electrons inside the atoms. This view has been confirmed, in so far as it applies to solids, by the discovery that the frequencies of the reststrahlen can be roughly computed from the elastic constants of the crystals ${ }^{3}$ and seems to be generally accepted by the workers in this field.

Nearly twenty-five years ago Lord Rayleigh ${ }^{4}$ pointed out that if an oscillator which at rest emits and absorbs light of frequency $\nu_{0}$, rotates with the frequency $\nu_{r}$ about an axis perpendicular to the direction of vibration, the emitted or absorbed light should be divided between the frequencies $\nu_{0}+\nu_{r}$ and $\nu_{0}-\nu_{r}$, and remarked that the absence of a corresponding broadening of the spectrum lines due to the molecular rotations constituted an outstanding difficulty in the theory of the

I Niels Bjerrum, Nernst Festsch1ift, Halle, I9I2.

2 P. Drude, Ann. d. Phys. (4), I4, p. 677, I904.

${ }^{3}$ Cf., for example, E. Madelung, Nachrichten d. kgl. Ges. d. W. z. Göttingen (Math. Phys. K1.), 20, p. II, I909; A. Einstein, "La Théorie du Rayonnement et les Quanta" (Proceedings of the First Solvay Conference), Paris, I9I2, p. 4I3; W. Dehlinger, Phys. Zeit., I5, p. 276 , I9I4.

${ }^{4}$ Lord Rayleigh, Phil. Mag. (5), 24, p. 410, I892. 
breadth of these lines. Bjerrum (1. c.) has called attention to the fact that in the infra-red absorption spectra of gases a broadening of the magnitude to be expected from the molecular rotations does occur. $\mathrm{He}$ explains the sharpness of the lines in other parts of the spectrum by assuming, first, that unlike the infra-red bands the lines in the visible and ultra-violet are due to the vibrations of electrons inside the atoms, and second, that the atoms do not rotate with angular velocities comparable with those of the molecules.

Consider the infra-red absorption of a diatomic gas like $\mathrm{HCl}$. If Drude's assumption is correct there should be but one absorption band (due to the vibration of the two atoms along their line of centers). It may be assumed that the rotation takes place only about axes perpendicular to the line of centers. If the distribution of angular velocities were in accordance with the classical statistical mechanics the band should be a doublet the maxima of which correspond to the most probable angular velocity of the molecules at the temperature in question. As a matter of fact the infra-red absorption spectrum of $\mathrm{HCl}$ does consist of just such a doublet, the maxima being at $3.40 \mu$ and $3.55 \mu$ at room temperature. From the separation of the maxima Bjerrum has made a rough computation of the moment of inertia of the molecule in the following manner. ${ }^{1}$ Let $\lambda^{\prime}$ and $\lambda^{\prime \prime}$ be the wave-lengths of the two maxima. Then if $\nu_{r}$ is the corresponding frequency of molecular rotation

and

$$
\nu_{0}+\nu_{r}=c / \lambda^{\prime}
$$

Consequently,

$$
\nu_{0}-\nu_{r}=c / \lambda^{\prime \prime} \text {. }
$$

$$
2 \nu_{r}=c\left(\frac{\mathrm{I}}{\lambda^{\prime}}-\frac{\mathrm{I}}{\lambda^{\prime \prime}}\right) .
$$

Identify this frequency with that corresponding to the average energy of molecular rotation. Then, if $J$ is the moment of inertia of the molecule,

or

$$
2 \pi^{2} \nu_{r}^{2} J=R T / N,
$$

$$
J=\frac{R T}{2 \pi^{2} \nu_{r}^{2} N},
$$

where $R$ is the gas constant and $N$ is the number of molecules in a gram molecule. Bjerrum assumes the values

$$
R=0.83 \times 10^{8}, \quad N=65 \times 10^{22}
$$

and for $T=29 \mathrm{I}^{\circ}$ computes

$$
J=5.4 \times 10^{-40} \mathrm{gm} . \mathrm{cm}^{2}
$$

${ }^{1}$ N. Bjerrum, Verh. d. D. Phys. Ges., I6, p. 640, I9r4. 
Assuming that the masses of the atoms are concentrated in the nuclei we can compute the distance between the atomic centers from the moment of inertia. ${ }^{1}$ The value so obtained is $\mathrm{I} .84 \times 10^{-8} \mathrm{~cm}$., which is in satisfactory agreement with the molecular "diameters" computed from the kinetic theory of gases. ${ }^{2}$

Double bands of this character are very frequent in the infra-red spectra of gases. ${ }^{3}$ In those cases tested the separation of the maxima varies with the temperature in accordance with Bjerrum's theory. ${ }^{4}$

Bjerrum predicted that the absorption bands of gases having more than two atoms per molecule would prove to be triplets, but his expectation has not been found to accord with the facts, as the absorption bands of water vapor and carbon dioxide, for instance, turn out to be doublets like those of the diatomic gases. Thus there is an outstanding difficulty with regard to the absorption of the more complex gases. However, this difficulty does not seem wholly insurmountable and the quantitative agreement of theory and experiment in the case of the diatomic gases leaves little doubt as to the fundamental soundness of Bjerrum's view.

The suggestion has been made by Lorent $z^{5}$ and Nernst ${ }^{6}$ that according to the older form of Planck's theory a molecule ought not to be capable of rotating with any angular velocity whatever, but only with such angular velocities as make the energy an integral multiple of $h \nu$. According to this hypothesis the possible frequencies of rotation are those which satisfy the equation

$$
\nu=\frac{n h}{2 \pi^{2} J},
$$

where $n$ is any integer Bjerrum has called attention to the fact that if this hypothesis is correct the absorption in the extreme infra-red (say beyond $50 \mu$ ) which we suppose is due to the direct action of the molecular rotations must be broken up into a series of equally spaced narrow bands, while the absorption bands in the near infra-red, due primarily to the atomic vibrations, must similarly be made up of a series of equally spaced fine lines which should appear when the bands are analyzed with an apparatus of sufficiently high resolving power. The predicted discon-

\footnotetext{
${ }_{1}^{1}$ Let $m_{1}$ and $m_{2}$ be the masses of the two atoms and let $r_{0}$ be the distance between the nuclei. Then $J=r_{0}^{2} m_{1} m_{2} /\left(m_{1}+m_{2}\right)$.

${ }^{2}$ D. A. Goldhammer (Verh. d. D. Phys. Ges., I6, p. 7I5, I9I4) estimates the "diameter" of the $\mathrm{HCl}$ molecule to be $3.0 \times \mathrm{IO}^{-8} \mathrm{~cm}$.

${ }^{3}$ W. Burmeister, Verh. d. D. Phys. Ges., I5, p. 589, I9I3.

${ }^{4}$ E. v. Bahr, Verh. d. D. Phys. Ges. I5, p. 7 Io and p. 73I, I9I3.

${ }^{5}$ H. A. Lorentz, "La Théorie du Rayonnement et les Quanta" (Proceedings of the First Solvay Conference), Paris, I9I2, p. 447.

${ }^{6}$ W. Nernst, Zeit. f. Elektroch., r7, p. 265, I9I r.
} 
tinuities in both parts of the infra-red spectrum have been found. ${ }^{1}$ The moment of inertia of the $\mathrm{HCl}$ molecule has been computed by means of (5) and the value obtained in this way has been found to agree with that computed from the separation of the maxima of the double band. Great as are the difficulties to which we are led by that form of the quantum theory which assumes that both absorption and emission take place by quanta, it would appear that there is little hope of escape from this essentially kinematical confirmation of that theory.

For further details regarding Bjerrum's theory and its experimental confirmation the reader is referred to the original papers.

In addition to (5) the following formula for the possible frequencies of rotation of a molecule on the basis of the quantum theory has been proposed by Ehrenfest. ${ }^{2}$

$$
\nu=\frac{n h}{4 \pi^{2} J} .
$$

(6) differs from (5) by a factor of two which comes from the fact that Ehrenfest assumes that the quantum for rotations is not $h \nu$ but $\frac{1}{2} h \nu$. He says that this substitution of $\frac{1}{2} h \nu$ for $h \nu$ can be justified from a very general point of view, but gives no further explanation. (Planck's generalization of the quantum theory to cover systems having several degrees of freedom ${ }^{3}$ also leads to (6)).

However that may be, Bjerrum has taken the very close agreement between the values of $J$ computed from (5) and from the separation of the maxima of the absorption bands doubled by the molecular rotations as evidence that (5) and not (6) is the correct formula. ${ }^{4}$ In this paper we propose to show that the approximation made by Bjerrum in computing the moment of inertia from the separation of the maxima of the double band in the infra-red spectrum of $\mathrm{HCl}$ was not so good as he supposed and that if the computation is correctly made it turns out that the resulting value of $J$ checks with that obtained from (6) and not with that obtained from (5).

We will also show that the shape of the carbon monoxide absorption band at $4.6 \mu$ is such as to give direct proof that the law of the distribution of angular velocities among the molecules at room temperature is that predicted by the classical statistical mechanics (except, of course, that the distribution is not continuous).

${ }^{1}$ Cf. N. Bjerrum, Nernst Festschrift, Halle, I9I2 and Verh. d. D. Phys. Ges., I6, p. 640, I9r4; A. Eucken,'Verh. d. D. Phys. Ges., I5, p. II59, I9r3; E. v. Bahr, 1. c.

2 P. Ehrenfest, Verh. d. D. Phys. Ges., 15, p. 45I, 19r3; A. Eucken, 1. c.

${ }^{3}$ M. Planck, Verh. d. D. Phys. Ges., I7, pp. 407 and 438, I9I5.

${ }_{4}^{4}$ N. Bjerrum, Verh. d. D. Phys. Ges., 16, p. 640, 1914. 
Deduction of the Value of the Moment of Inertia from the Separation of the Absorption Maxima.

The roughness of Bjerrum's approximation consisted in identifying the angular velocity corresponding to the maxima of the absorption doublet, $i$. e., the most probable angular velocity, with the angular velocity corresponding to the average energy. This makes a considerable difference in the case of the linear velocities of the molecules or of the angular velocities among molecules capable of rotating about three mutually perpendicular axes, but it makes a much greater difference if, as in the case of $\mathrm{HCl}$, the molecule has only two rotational degrees of freedom.

In what immediately follows it will be assumed that at ordinary room temperatures the distribution of angular velocities among the molecules is in accordance with the classical statistical mechanics, the justification for this hypothesis being given later. The molecule is treated as a pair of heavy particles rigidly joined together.

Let the masses of the atoms in a diatomic molecule be $m^{\prime}$ and $m^{\prime \prime}$. The positions of the nuclei with reference to a set of axes fixed in direction and having its origin at the center of mass of the $r$ molecule will be specified by the spherical coördi-

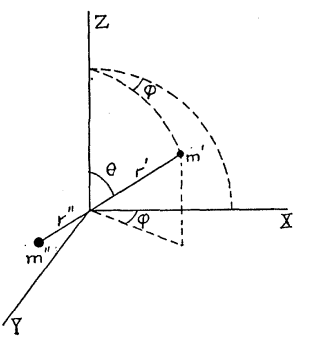

Fig. 1.

nates $r^{\prime}, r^{\prime \prime}, \theta, \varphi$ (Fig. I). Then the rotational energy is easily shown to be

$$
\boldsymbol{\epsilon}_{r}=\frac{1}{2}\left(m^{\prime} r^{\prime 2}+m^{\prime \prime} r^{\prime \prime 2}\right)\left(\dot{\theta}^{2}+\sin ^{2} \theta \cdot \dot{\varphi}^{2}\right)=(J / 2)\left(\dot{\theta}^{2}+\dot{\varphi}^{2} \sin ^{2} \theta\right) .
$$

$\dot{\theta}$ and $\varphi \sin \theta$ are the components of the angular velocity in and perpendicular to the $x y$ plane respectively. It is therefore convenient to denote them by $\omega^{\prime}$ and $\omega^{\prime \prime}$, so that

$$
\epsilon_{r}=\frac{1}{2} J\left(\omega^{\prime 2}+\omega^{\prime \prime 2}\right) \equiv \frac{1}{2} J \omega^{2} .
$$

Consider a Gibbs canonical ensemble of systems, each of which is made up of $\mu$ diatomic molecules. In the notation of Gibbs, in which the coördinates are denoted by $q$ 's and the momenta by $p$ 's, the chance that a system chosen at random will fall into any particular element of extension in phase,

is

$$
d p_{1}, \cdots d p_{n}, \quad d q_{1}, \cdots d q_{n},
$$

$$
d P=e^{(\psi-\epsilon) \Theta} d p_{1}, \cdots d p_{n}, \quad d q_{1}, \cdots d q_{n} .^{1}
$$

Here $\psi$ and $\Theta$ are constants, the latter being the modulus of the ensemble

${ }^{1}$ Cf. J. W. Gibbs, Elementary Principles in Statistical Mechanics, p. 33. 
(i.e., $R T / N) . \quad \epsilon$ is the energy corresponding to this element of extension in phase.

The phase of each molecule will be specified by five coördinates, viz., $\theta, \varphi$, and the coördinates of the center of mass, together with the corresponding momenta. The coördinates and momenta which specify the positions and velocities of the centers of mass may be eliminated by integrading over all possible values of these quantities. Denote the various molecules in any one system by the subscripts $\mathrm{I}, 2, \cdots \mu$. Then the chance that a system chosen at random will lie within the region

is

$$
d\left(p_{\theta}\right)_{1}, d\left(p_{\phi}\right)_{1}, d\left(p_{\theta}\right)_{2} \cdots d\left(p_{\phi}\right)_{\mu}, d \theta_{1} \cdots d \varphi_{\mu}
$$

where

$$
d P=C e^{(\psi-\epsilon) / \Theta} d\left(p_{\theta}\right)_{1} \cdots d \varphi_{\mu},
$$

and

$$
\left(p_{\theta}\right)_{i}=\frac{\partial \epsilon}{\partial \theta_{i}}=J \dot{\theta}_{i}=J \omega_{i}^{\prime}
$$

$$
\left(p_{\phi}\right)_{i}=\frac{\partial \epsilon}{\partial \varphi_{i}}=J \sin ^{2} \theta_{i} \cdot \dot{\varphi}_{i}=J \omega_{i}^{\prime \prime} \sin \theta .
$$

In order to obtain the law of the distribution of velocities from (Io) it is necessary to change the independent variables from $\left(p_{\theta}\right)_{i},\left(p_{\phi}\right)_{i}$, $\theta_{i}, \varphi_{i}$, to $\omega_{i}{ }^{\prime}, \omega_{i}{ }^{\prime \prime}, \theta_{i}, \varphi_{i}$. We have

$$
\begin{aligned}
d\left(p_{\theta}\right)_{i} d\left(p_{\phi}\right)_{i} d \theta_{i} d \varphi_{i} & =\left|\frac{D\left[\left(p_{\theta}\right)_{i}\left(p_{\phi}\right)_{i}, \theta_{i}, \varphi_{i}\right]}{D\left[\omega_{i}{ }^{\prime \prime}, \omega_{i}{ }^{\prime}, \theta_{i}, \varphi_{i}\right]}\right| d \omega_{i}{ }^{\prime \prime} d \omega_{i}{ }^{\prime} d \theta_{i} d \varphi_{i} \\
& =J^{2} \sin \theta_{i} d \omega_{i}{ }^{\prime \prime} d \omega_{i}{ }^{\prime} d \theta_{i} d \varphi_{i} .
\end{aligned}
$$

Then (I0) becomes

$$
d P=C e^{\psi / \Theta} J^{2 \mu} \sin \theta_{1} \cdots \sin \theta_{\mu} e^{-(\epsilon / \Theta)} d \omega_{1}{ }^{\prime \prime} \cdots d \omega_{1}{ }^{\prime} \cdots d \omega_{\mu}{ }^{\prime} d \theta_{1} \cdots d \varphi_{\mu} .
$$

Let $\epsilon^{\prime}$ denote that part of the energy not due to the rotations. Then

$$
\epsilon=\sum_{i=1}^{\mu} \frac{1}{2} J\left(\omega_{i}{ }^{2}+\omega_{i}{ }^{\prime 2}\right)+\epsilon^{\prime}=\sum_{i=1}^{\mu} \frac{1}{2} J \omega_{i}{ }^{2}+\epsilon^{\prime} .
$$

The chance that in a system chosen at random the $\nu$ th molecule will have an angular velocity in the elementary region $d \omega^{\prime} d \omega^{\prime \prime}$ is

$$
\begin{aligned}
C e^{-J / 2 \Theta\left(\omega^{\prime 2}+\omega^{\prime \prime 2}\right)} d \omega_{\nu}^{\prime} d \omega_{\nu}^{\prime \prime}\left[\int_{\text {values }}^{\text {all }}\right. & \cdots \int e^{1 / \Theta\left\{\psi-\Sigma_{2}^{\prime \prime} J \omega^{2}-\epsilon^{\prime}\right\}} \\
& \left.\times J^{2 \mu} \sin \theta_{1} \cdots \sin \theta_{\mu} d \omega_{1}^{\prime \prime} \cdots d \varphi_{\mu}\right],
\end{aligned}
$$

where $\Sigma^{\prime}$ denotes summation over all the molecules but one. The integral is independent of $\nu$ and of the region element under consideration. Hence it may be treated as a constant. Denote the product of $C$ into this integral by $C^{\prime}$. Then the most probable value of the total number 
of molecules having their velocities in the elementary region $d \omega^{\prime} d \omega^{\prime \prime}$ is

$$
\mu C^{\prime} e^{-(J / 2 \Theta)\left(\omega^{2}+\omega^{\prime \prime 2}\right)} d \omega^{\prime} d \omega^{\prime \prime} .
$$

The numerical value of $C^{\prime}$ may be determined from the equation

It is

$$
\mu=\mu C^{\prime} \iint e^{-(J / 2 \Theta)\left(\omega^{\prime 2}+\omega^{\prime \prime 2}\right)} d \omega^{\prime} d \omega^{\prime \prime} .
$$

$$
C^{\prime}=\frac{J}{2 \pi \theta} .
$$

If $\omega^{\prime}$ and $\omega^{\prime \prime}$ be regarded as rectangular coördinates the corresponding polar coördinates are

and

$$
\omega \equiv \sqrt{{\omega^{\prime 2}+\omega^{\prime \prime 2}}^{2}}
$$

$$
\chi=\tan ^{-1}\left(\frac{\omega^{\prime}}{\omega^{\prime \prime}}\right) \text {. }
$$

Then the region element $d \omega^{\prime} d \omega^{\prime \prime}$ may be replaced by $\omega d \omega d \chi$. The most probable value of the number of molecules whose resultant angular velocity lies between $\omega$ and $\omega+d \omega$ is

$$
\frac{\mu J}{\Theta} e^{-\frac{J \omega}{2 \Theta}} \cdot \omega d \omega .
$$

(I5) is an expression of the distribution law for angular velocities in a diatomic gas. The corresponding expression for the law of the distribution of linear velocities is

$$
\sqrt{\frac{2 m^{3}}{\pi \Theta^{3}}} e^{-\frac{m v^{2}}{2 \Theta}} \cdot v^{2} d v
$$

We desire to compute from (I5) the ratio of the most probable angular velocity to the angular velocity corresponding to the average energy. The most probable angular velocity is that for which

$$
\frac{d}{d \omega}\left(\omega e^{-\frac{J \omega^{2}}{2 \Theta}}\right)=e^{-\frac{J \omega^{2}}{2 \Theta}}-\frac{J \omega^{2}}{\Theta} e^{-\frac{J \omega^{2}}{2 \Theta}}=0 .
$$

Let $\Omega$ be a root of this equation. Then

The average energy of rotation is

$$
\Omega= \pm \sqrt{\frac{\Theta}{J}}
$$

$$
\bar{\epsilon}_{\omega}=\frac{J}{\Theta} \int_{0}^{\infty} e^{-\frac{J \omega^{2}}{2 \Theta}} \cdot \frac{J \omega^{2}}{2} \cdot \omega d \omega=\theta .
$$

Hence

$$
\Omega= \pm \sqrt{\frac{\overline{\epsilon_{\omega}}}{J}}= \pm \sqrt{\frac{R T}{J N}}
$$


Let $\nu_{r}$ denote the corresponding frequency. Then we have

$$
J=\frac{R T}{4 \pi^{2} \nu_{r}^{2} N}
$$

instead of (4). Accordingly the moment of inertia of the $\mathrm{HCl}$ molecule is

$$
J=2.7 \times \mathrm{IO}^{-40} \mathrm{gm} . \mathrm{cm}^{2}
$$

This checks with (6) exactly as the value computed by Bjerrum checked with $(5) \cdot{ }^{1}$

Rotation Frequencies of the $\mathrm{HCl}$ Molecule.

\begin{tabular}{l|c|c}
\hline Observed E. v. Bahr. & $n$. & Computed by (6). \\
\hline $0.745 \times 10^{12}$ & 1 & $0.6 \times 10^{12}$ \\
1.395 & 2 & 1.2 \\
2.015 & 3 & 1.8 \\
2.62 & 4 & 2.4 \\
3.20 & 5 & 3.0 \\
3.68 & 6 & 3.6 \\
4.08 & 7 & 4.2 \\
\hline
\end{tabular}

Experimental Proof of the Velocity Distribution Law.

As a check on our theory of the structure of the infra-red absorption bands it is of interest to compare the form of the absorption curve predicted by (I5) with that found experimentally by Burmeister (1. c.), who has investigated the infra-red absorption spectra of a number of the simpler gases with great care.

We have already called attention to the fact that a molecule whose frequency of oscillation is $\nu_{0}$ and whose frequency of rotation is $\nu_{r}$ will emit or absorb light of the frequencies $\nu_{0}+\nu_{r}$ and $\nu_{0}-\nu_{r}$. Let us call these the effective frequencies of the molecular vibration. We assume that the ability of a molecule to absorb light is not affected by its rotary motion. Then the absorption coefficient of the gas in the frequency interval between $\nu_{0}$ and $\nu+\Delta \nu$ should be proportional to the number of molecules which have effective frequencies in that interval. Consequently if (I5) gives the distribution law correctly the curve obtained when the absorption coefficient is plotted against frequency should be of the form

$$
\alpha= \pm a e^{-b\left(\nu-\nu_{0}\right)^{2}}\left(\nu-\nu_{0}\right) .
$$

Of course the discontinuities which have been found in the absorption bands show that (I9) cannot be strictly accurate. What we may really expect is a series of equally spaced narrow absorption bands having such

1 The following table (taken from Bjerrum, 1. c.) gives the observed rotation frequencies together with the values computed by means of (6) and (I8). The small systematic error is presumably due to the variation of $J$ with the frequency of rotation. 
relative intensities that a curve of the form (I9) is their envelope. The number of molecules whose effective frequencies lie in the interval between $\nu$ and $\nu+\Delta \nu$ will be proportional to

$$
\left(\nu-\nu_{0}\right) e^{-b\left(\nu-\nu_{0}\right)^{2}}
$$

only if that interval is large enough to cover a number of these elementary bands or lines. However, the absorption curve obtained with an apparatus whose resolving power is insufficient to separate these elementary bands should be of the form (I9).

The curves given by Burmeister (cf. Fig. 2) show the total observed absorption in tubes of $20 \mathrm{~cm}$. or of $40 \mathrm{~cm}$. length plotted as a function of the wave-length. The two pairs of short vertical lines indicate the wavelength interval subtended by the slit. We have first to compute the values of the absorption coefficient corresponding to the various observed total absorptions. This would be easy enough if the various bundles

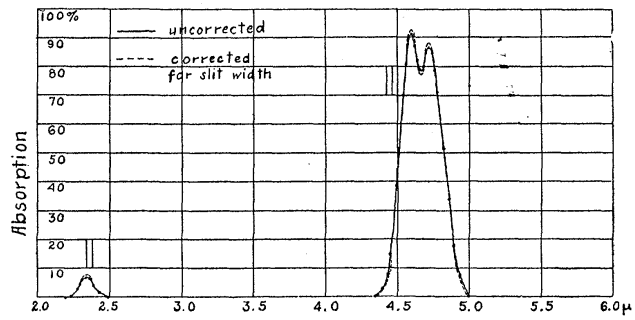

Infra-red Absorption of Carbon Monoxide (after Burmeister).

Fig. 2.

of rays examined were sufficiently homogeneous so that the absorption coefficient for all frequencies in any one bundle could be regarded as constant. Let $I_{0}$ denote the initial intensity of such a bundle and let $I$ denote its intensity after passing through $L$ centimeters of absorbing gas. Then under the ideal conditions specified

$$
I=I_{0} e^{-a L}
$$

and the absorption coefficients are proportional to the values of $\log I / I_{0}$. But in Burmeister's experiments the resolving power of the spectrometer was not sufficient to separate the band into the lines which compose it. Consequently the light passing through the slit at any one time was not of sufficient homogeneity so that all parts of it were absorbed with equal readiness. Under these conditions the total energy of the beam does. not vary according to (20). In fact the discontinuous character of the infra-red absorption bands was first proved by observing the failure of the absorption law (20). ${ }^{1}$

${ }^{1}$ E. v. Bahr, Verh. d. D. Phys. Ges., I5, p. 7ro, 1913. 
We have data showing the actual absorption law for the complex beams under consideration only in the case of one of the diatomic gases whose absorption bands were studied by Burmeister, viz., CO. In Fig. 3 we reproduce an experimental curve due to Dr. v. Bahr (1. c.) showing the relation between the total absorption in per cent. and the partial pressure of the absorbing carbon monoxide. This is of the same form as the curve connecting the absorption in per cent. with the length of tube for constant partial pressure. The dotted portion of the curve is

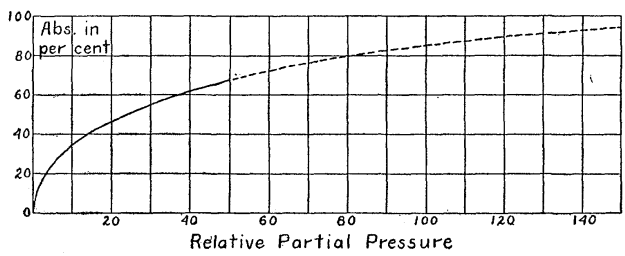

Fig. 3.

an extrapolation which should be fairly accurate as it was obtained with the help of an asymptote (the roo per cent. absorption line). The initial slope of the curve is a measure of the average absorption coefficient of the different wave-lengths in the beam. Dr. v. Bahr reports that this curve is of the same form whether the slit used is comparatively narrow or wide enough to take in the whole band. We conclude that it would be the same for a narrow slit set to cover any portion of the band. By the statement that two or more absorption curves are of the same form we mean that the curves can be made to coincide by a suitable change of the scales which indicate the lengths of the absorption paths. Then to get the absorption curve for one frequency interval from that for another, we must multiply the abscissæ of the given curve by a number so chosen that the ratios of the absorptions from the two intervals in the same length of tube shall check with the corresponding ordinates of Burmeister's curve. The initial slopes or absorption coefficients for different curves will be inversely proportional to these multipliers. It follows that the absorption coefficients corresponding to two different values of the total absorption are directly proportional to the abscissæ corresponding to these absorptions on Dr. v. Bahr's curve.

In this manner the writer has obtained a curve of absorption coefficients from the curve of total absorptions of the double band at $4.6 \mu$ given by Burmeister. The reader will observe (Fig. 2) that the latter curve is unsymmetrical, the absorption being greater on the high frequency side. This lack of symmetry is much greater in the curve of absorption coefficients and is at present wholly unexplained. ${ }^{1}$ The absorption coefficients

${ }^{1}$ We should expect a difference proportional to the difference in the squares of the effective frequencies but this is small. 
of symmetrically situated points have therefore been averaged. The resulting average absorption coefficient plotted against the corresponding angular velocity (in arbitrary units) is shown in Fig. 4, Curve $a$. Curve $b$ is plotted from the equation

$$
y=299.5 x e^{-x^{2}}
$$

(cf. equation 19). Curve $c$ shows the distribution law predicted by

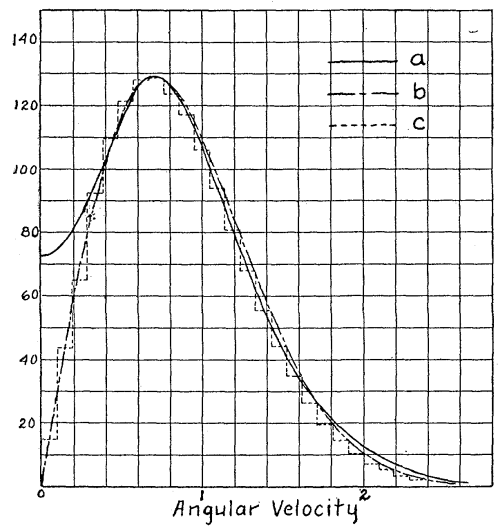

Fig. 4.

Planck (1. c., p. 4) on the basis of his later form of the quantum theory. (Curve $c$ could be made to fit the experimental curve somewhat better by a slightly different choice of moment of inertia.) The agreement between the experimental and theoretical curves seems to the writer remarkably good under the circumstances. The discrepancy at zero angular velocity is undoubtedly due largely to the wide slit used. The values of the total absorption have been corrected for slit width according to Runge's formula but this formula would not apply at this point where the change in the absorption coefficient is discontinuous.

The agreement between the experimental and theoretical curves justifies the assumption of the distribution law (I5) in computing the moment of inertia of the $\mathrm{HCl}$ molecule. Apparently the true distribution law is to be obtained by some small modification of Planck's extension of the quantum theory to systems having several degrees of freedom so as to adapt that extension to the hypothesis of discontinuous absorption.

\section{SUMmary.}

We have developed a formula for the distribution of angular velocities among diatomic gas molecules on the basis of the classical statistical mechanics and have shown that this formula is in agreement with the 
observed shape of the band at $4.6 \mu$ in the infra-red absorption spectrum of carbon monoxide (the only diatomic gas for which the necessary data are available).

On the basis of this distribution law we have shown that the expression for the moment of inertia of a diatomic molecule in terms of the frequency of rotation $\nu_{r}$ corresponding to the maxima of a Bjerrum double band is

and not

$$
J=\frac{R T}{4 \pi^{2} \nu_{r}^{2} N}
$$

$$
J=\frac{R T}{2 \pi^{2} \nu_{r}^{2} N} .
$$

We have shown that this new formula for the moment of inertia checks with Ehrenfest's assumption that the quantum for the rotational energy should be $\frac{1}{2} h \nu$ instead of $h \nu$.

JefFerson Physical Laboratory, HaRVARD University,

August 7, r916. 